\title{
Comment
}

\section{Agrotóxicos em água para o Consumo Humano}

\author{
Manuel Gonçalves de Almeida Neto ${ }^{1}$; Rosilaine Barbosa Silva Reis. ${ }^{2}$
}

Resumo: Os agrotóxicos compõe uma variedade de substancias químicas, que são utilizados pelo ser humano, mas estes podem se apresentar como contaminantes do ambiente e com isso trazer riscos aos seres vivos, e o uso indiscriminado gera consequências graves no que diz respeito ao contato de trabalhadores com tais substâncias. E entre as formas de contaminação dos agrotóxicos, pode-se citar a água usada para irrigação e manejo da agricultura como uma fonte que é facilmente contaminada, o que é um risco para o meio ambiente e para a saúde pública. Diante deste contexto, este estudo teve por objetivo fazer um levantamento dos estudos que analisam águas contaminadas com agrotóxico. A partir disto, por meio da discussão da temática com a pesquisa bibliográfica e documental fundamentada no método qualitativo, demonstra-se que a água e a sua condição é fator essencial para a segurança dos alimentos e saúde do consumidor e por isso é necessário a vistoria da qualidade da água sempre que utilizada para consumo humano, além da análise de resíduos de agrotóxicos nas mesmas.

Palavras- chave: Água, Agrotóxicos, Consumo humano.

\section{Agrochemicals in water for human consumption}

\begin{abstract}
Pesticides make up a variety of chemicals, which are used by human beings, but these may present as environmental contaminants and thus bring risks to living beings, and the indiscriminate use generates serious consequences with regard to contact workers such substances. And among the forms of contamination of pesticides, you can mention the water used for irrigation and management of agriculture as a source that is easily contaminated, which is a risk to the environment and to public health. Given this context, this study aimed to survey the studies that analyze water contaminated with pesticides. From this, by the thematic discussion with the bibliographic and documentary research based on the qualitative method, it is shown that water and your condition is an essential factor for food safety and consumer health and therefore the survey is required water quality when used for human consumption, beyond the pesticide residues in the same analysis.
\end{abstract}

Key word: Water, Agrochemicals. Human consumption.

\section{Introdução}

O solo e a água são recursos naturais de extrema importância para a sobrevivência do ser humano, e neste contexto destaca-se a utilização de técnicas na produção agrícola que dispõe de uma grande variedade de substâncias sintéticas que são colocadas no meio ambiente, dentre elas os agrotóxicos são um alerta por gerarem risco ambiental se manejados de forma errada.

\footnotetext{
${ }^{1}$ Faculdade Independente do Nordeste - FAINOR. E-mail: nelibce1 @ hotmail.com.

${ }^{2}$ Graduada em Nutrição pela Faculdade de Tecnologia e Ciências (FTC). Especialista em Nutrição Clínica e Funcional pela Faculdade de Tecnologia e Ciências (FTC - 2014) de Vitória da Conquista - Bahia. Especialista em Processo Tecnológico e Controle de Qualidade de Alimentos pela Faculdade Independente do Nordeste (FAINOR - 2016). Atua como preceptora do estágio em indústria de alimentos, no curso de Farmácia da Faculdade Independente do Nordeste - FAINOR no município de Vitória da Conquista-Bahia.
} 
Id on Line Revista Multidisciplinar e de Psicologia

Id on Line Multidisciplinary and Psycology Journal

Diante disso, a qualidade da água torna-se uma preocupação latente, e o Ministério da Saúde propõe os procedimentos de controle e de vigilância da qualidade da água potável, relacionando a seu padrão de potabilidade (BRASIL, 2011).

$\mathrm{Na}$ avaliação da qualidade microbiológica da água pode ser realizada por meio da análise de indicadores de contaminação fecal e biológicos (BUZANELLO et al., 2008).

O uso intensivo de agrotóxicos no país tem gerado um problema com relação a segurança da qualidade da água, pois tais sustâncias trazem riscos potenciais ao ambiente e aos seres humanos e quando afeta a água para consumo humano gera problemáticas com graves efeitos. Salientando que existe Portaria do Ministério da Saúde $n^{\circ}$ 518/2004 que trata da potabilidade da água e considera 22 agrotóxicos (FERNANDES NETO; SARCINELLI, 2009).

Muitos agrotóxicos, a exemplo dos inseticidas, fungicidas, herbicidas e nematicidas, detém consigo um princípio ativo tóxico, normalmente com potencial poluidor em águas e isso gera impacto ambiental, pois os níveis de componentes bióticos do ecossistema, afetam a funcionalidade e sustentabilidade deste (STEFFEN, et al, 2011).

Diante destas problemáticas este estudo tem por objetivo alertar sobre a importância da água para os seres vivos e seu uso racional na atividade agrícola., enfatizando os meios de contaminação da água causados pelos agrotóxicos na agricultura, para por fim relatar as formas de prevenção e tratamento de agrotóxicos na água utilizada pelos produtores de modo a conscientizar os produtores sobre os males que os agrotóxicos podem causar à saúde pública.

Justifica-se estudo pela importância de destacar a segurança da água e do uso consciente de agrotóxicos na agricultura, evitando contaminação, e sendo esta discussão relevante para conscientização humana a respeito desta temática.

\section{Metodologia}

A presente pesquisa é qualitativa e, caracteriza-se por discutir hipóteses, de modo geral, utilizando-se de levantamento bibliográfico. Para Lakatos e Marconi (2010) responde a questões particulares, trabalhando com um universo de significados, correspondendo a um espaço mais profundo de tais relações. 
Para a realização desta pesquisa foi feito um estudo de revisão bibliográfica, caracterizada por ser desenvolvida com base em material já elaborado de revisões da literatura publicadas, artigos científicos, trabalhos publicados em revistas e periódicos, livros e consultas via internet sobre a temática abordada. A pesquisa bibliográfica é de grande relevância à ciência. Nessa linha de raciocínio, Minayo (2008, p. 48) destaca: “a pesquisa bibliográfica é meio de formação por excelência. Como trabalho científico original, constitui a pesquisa propriamente dita na área das ciências humanas".

Segundo Lakatos e Marconi (2010), a pesquisa bibliográfica trata do levantamento, seleção e documentação de toda bibliografia previamente publicada sobre o assunto que está sendo pesquisado em livros, revistas, jornais, artigos, publicações avulsas e impressos.

Para Minayo (2008), este tipo de pesquisa é desenvolvida por meio de fontes já existente ou publicadas, sendo considerado o livro como principal fonte, tendo como objetivo de redefinir um problema, proporcionar uma melhor visão deste ou torná-lo mais específico, considerando que estes são amplos e pouco esclarecidos.

\section{Comentários á partir da Literatura}

Consideram-se utilização de água imprópria ao consumo humano que pode comprometer a saúde humana, levando riscos aos consumidores. Neste contexto, a potabilidade de água deve ser considerada, e quando a qualidade da água é ameaçada por substâncias agrotóxicas ela não fica aceitável para o consumo.

O Ministério da saúde em 2013 publicou um Boletim Epidemiológico Secretaria de Vigilância em Saúde, e neste destaca que o Brasil é o maior consumidor mundial de agrotóxicos e enfatiza os riscos relacionados à exposição humana a substâncias químicas. Este estudo averiguou seis artigos científicos que abordavam a temática vigente, conforme tabela a seguir: 
Id on Line Revista Multidisciplinar e de Psicologia

Id on Line Multidisciplinary and Psycology Journal

Tabela 1: Distribuição referente aos artigos científicos que tratam sobre o tema, conforme Base de Dados.

\section{Base de dados}

Base de Dados

\section{Descritores}

Agrotóxico em água

LILACS Consumo humano

Efeitos

Contaminação de agrotóxico

PUBMED

Contaminação de água

SCIELO Contaminação de água

Agrotóxicos
Aquíferos contaminados

\section{Total de artigos}

encontrados

$\mathbf{N}$

$\%$

01

16,7

01

16,7

$04 \quad 66,6$

Total

06

Fonte: Dados da Pesquisa, 2016.

Neste contexto, analisa-se a utilização de seis artigos, onde houve uma maior prevalência de artigos publicados na SCIELO, com $66,6 \%$ de artigos sobre os temas selecionados publicados nesta base de dados, e 16,7\% do PUBMED e 16,7\% do LILACS e os artigos abordaram os seguintes descritores: Agrotóxico em água, Consumo humano, Efeitos, Contaminação de agrotóxico, Aquíferos, Contaminação de água.

Os artigos selecionados estão abaixo listados conforme autores, título e desfecho do estudo:

Tabela 2: Artigos Selecionados. Brasil, 2016.

\section{Artigos selecionados}

Autores

Titulo

Desfecho

Steffens et al (2011)

Casali

(2008)

\section{Moreira et al} (2012)

\section{Contaminação do solo e da água} pelo uso de agrotóxicos.

Qualidade da água para consumo humano ofertada em escolas e comunidades rurais da região central do Rio Grande do Sul.

Contaminação de águas superficiais e de chuva por agrotóxicos em uma região do estado do Mato Grosso.
Efeitos da contaminação

Destaca a água fora dos padrões de potabilidade.

Deterioração da água por agrotóxicos em concentrações superiores às recomendadas 


\begin{tabular}{ccc} 
Alves e Silva (2003) & $\begin{array}{c}\text { Avaliação de ambientes } \\
\text { contaminados por agrotóxicos } \\
\text { Qualidade sanitária de águas de } \\
\text { Farache Filho, Neto } \\
\text { (2004) }\end{array}$ & $\begin{array}{c}\text { Análise macro sobre como a } \\
\text { contaminação gera danos }\end{array}$ \\
$\begin{array}{c}\text { Mortas em municípios da região de } \\
\text { Araraquara -SP. } \\
\text { et al (2010) }\end{array}$ & $\begin{array}{c}\text { Repercussões da água contaminada } \\
\text { com agrotóxicos }\end{array}$ \\
& $\begin{array}{c}\text { Resíduos de agrotóxicos na água } \\
\text { Estado do Rio Grande } \\
\text { do Sul, Brasil. }\end{array}$ & $\begin{array}{c}\text { Contaminação de água } \\
\text { por agrotóxicos }\end{array}$ \\
\hline Fonte: Base de Dados PubMed, LILACS e SCIELO (2016).
\end{tabular}

Estudos frequentes demonstram a poluição da água de diversos meios, e no caso de agrotóxicos a preocupação é frequente devido as problemáticas que tal problema pode trazer para o ser humano.

Neste sentido, o estudo feito por Steffens et al. (2011), que trata da contaminação do solo e da água pelo uso de agrotóxicos destaca a respeito dos efeitos do agrotóxico em água para consumo humano, destacando que além de contaminar o ar, o solo, as águas superficiais e subterrâneas, os agrotóxicos causam problemas à saúde humana. Faz um alerta para o fato de a contaminação da água com resíduos de agrotóxicos provoca efeito em todos os seres vivos. Pela necessidade de existir o uso destes para o plantio esse autor relata que o uso deve ser realizado de uma forma racional e responsável, protegendo a saúde humana e o meio ambiente.

Casali (2008), confirma tal preocupação em seu estudo e enfatiza que a ação humana é responsável pela contaminação de mananciais hídricos e para evitar a poluição dos recursos naturais deve existir um alerta grande com relação ao uso de agrotóxicos, para isso deve-se trabalhar a conscientização daqueles que fazem manejo destas sustâncias a exemplo dos agricultores com a relevância de implementar tais cuidados.

Moreira et al (2012) mostra em seu estudo que a o uso de agrotóxicos afeta a biota e a contaminação das águas de córregos e da água de chuvas indica que produtos tóxicos já contaminaram a atmosfera gerando cada vez mais piores impactos ou riscos sobre a saúde ambiental. Os resultados deste estudo destacam que a deterioração da água potável causada pela 
Id on Line Revista Multidisciplinar e de Psicologia

Id on Line Multidisciplinary and Psycology Journal

volatilização de agrotóxicos usados nos processos agrícolas e sua precipitação através das chuvas, gera impactos de difícil mensuração e que atingirão gerações futuras, por isso são necessárias estratégias de vigilância em saúde ambiental neste país.

Alves e Silva (2003) destacaram de igual maneira que a permanência dos agrotóxicos no meio ambiente poluem a água e as suas propriedades físico-químicas dos contaminantes geram problemáticas na veiculação pelo ar, e, acresce a necessidade de implementação de normas de segurança, que elevem a preocupação com os riscos dos agrotóxicos. Muitas doenças como câncer, distúrbios reprodutivos podem ser manifestados por uso de água contaminada e afeta a biodiversidade da fauna aquática e consequentemente da qualidade de vida local dos seres vivos que utilizam da água ou que a ela estão expostos.

Farache Filho e Gustavo Neto (2004) destacam que a água quando contaminada com agrotóxicos afetam a saúde e a aceitação para consumo. Por isso é importante primar pela definição de um padrão para contaminantes, com valores-guia preconizados pela lei em prol de evitar contaminações e punir os agentes agressores do meio ambiente.

Normas atuais tidas como Diretivas consideram os parâmetros 'Agrotóxico' e 'Agrotóxico total', cujos VMP são, respectivamente, $0,1 \mu \mathrm{g} / \mathrm{L}$ e $0,50 \mu \mathrm{g} / \mathrm{L}$. No qual o VMP para o parâmetro Agrotóxico é aplicado para cada substância à exceção de Aldrin, Dieldrin, Heptacloro e Heptacloro epóxido, cujos VMP são o mesmo: 0,03 $\mu \mathrm{g} / \mathrm{L}$. Com relação ao agrotóxico total, a soma das concentrações de todas as substâncias não deve ser maior que 0,50 $\mu \mathrm{g} / \mathrm{L}$.

Marchesan et al (2010) demonstram uma preocupação com a água contaminada com agrotóxicos, sobretudo por causa do risco à saúde humana (ingestão oral), e para isso deve-se entender os efeitos que tais substâncias geram ao ser humano, analisando estudos sobre a toxicidade e com relação a preocupação com os níveis de exposição.

Por fim, nota-se que tais estudos discutiram aspectos relacionados às intoxicações por agrotóxicos gerados pela poluição em água, e no fomentando os desafios para evitar este problema. Incentiva-se deste modo mais estudos epidemiológicos, analisando os efeitos relacionados a exposição de seres humanos com a água contaminada por agrotóxicos. Isso pelo fato de que os efeitos gerados a saúde humana pelo consumo de água contaminada por agrotóxicos vão variar conforme o princípio ativo envolvido. Estudos citam problemas no 
fígado e no sistema nervoso central, no sistema cardiovascular e reprodutivo, e ainda outras doenças que afetam olhos, rins, baço, gera anemia e aumento do risco de desenvolver câncer.

Soares et al (2003) e Veiga et al (2006) de igual modo enfatizam a preocupação com a qualidade da água como forma de evitar problemas deste tipo, e destacam a preocupação com o uso de agrotóxicos e a relevância de normas que tratem deste assunto. Alertam para o fato de que a maioria das pessoas que lidam com estes produtos não leem o rótulo de forma adequada ou não saem lidar com as instruções de forma correta. Os agricultores e manipuladores não apresentam conhecimento sobre os produtos de forma adequada e ainda não há uma fiscalização efetiva do serviço quanto ao uso e aplicação de produtos deste tipo, necessitando de uma maior fiscalização e acompanhamento técnico e no controle de agrotóxicos, levando em consideração os cuidados com a água e a contaminação, promovendo qualidade de vida, de saúde e ao meio ambiente.

\section{Conclusão}

Nesse sentido, o estudo demonstrou que muitos trabalhos contemplam a preocupação atual com o ambiente relacionado a água e agrotóxicos, e quando este recurso natural é contaminado, trazendo efeitos negativos para a saúde humana, de modo a gerar problemas de saúde que podem levar até a morte.

Um dado alarmante observado foi que a contaminação acontece pelo fato de não haver conhecimento suficiente para lidar com o produto e ainda pelo fato de não haver uma fiscalização efetiva a respeito às dificuldades encontradas no uso de sustâncias deste tipo.

\section{Referências}

ALVES, SR., SILVA, JJ. Avaliação de ambientes contaminados por agrotóxicos. In: PERES, F., and MOREIRA, JC., orgs. É veneno ou é remédio?: agrotóxicos, saúde e ambiente [online]. Rio de Janeiro: Editora FIOCRUZp. 137-156, 2003. 
Id on Line Revista Multidisciplinar e de Psicologia

Id on Line Multidisciplinary and Psycology Journal

BOHNER. Tanny Oliveira Lima; ARAUJO. Luiz Ernani Bonesso; NISHIJIMA. Toshio. O impacto ambiental do uso de agrotóxicos no meio ambiente e na saúde dos trabalhadores rurais. Revista Eletrônica do Curso de Direito da UFSM. 2010.

BRASIL. Ministério da Saúde. Portaria n. ${ }^{\circ}$ 2914, de 12 de dezembro de 2011. Diário Oficial da União, Brasília, 14 dez. 2011, Seção 1, p.39-46.

Ministério da Agricultura, Pecuária e Abastecimento. Secretaria De Defesa Agropecuária. Instrução Normativa No 62, de 26 de Agosto de 2003.

BUZANEllo, E. B., MARTinhago, M. W., AlMEIDA, M. M., PINTO, F. G. S. Determinação de Coliformes Totais e Termotolerantes na Água do Lago Municipal de Cascavel, Paraná. Revista Brasileira de Biociências, v. 6, supl. 1, p. 59-60. 2008.

CASALI. Carlos Alberto. Qualidade da água para consumo humano ofertada em escolas e comunidades rurais da região central do Rio Grande do Sul. Dissertação (Mestrado). Santa Maria, RS, Brasil. 2008.

FARACHE FILHO, A., GUSTAVO NETO, F. Qualidade sanitária de águas de irrigação de hortas em municípios da região de Araraquara -SP. Relatório PIBIC-Programa Institucional de Bolsas de Iniciação Cientifica. Faculdade de Ciências Farmacêuticas de Araraquara, UNESP, 2004.

FERNANDES NETO. Maria de Lourdes. SARCINELLI. Paula de Novaes. Agrotóxicos em água para consumo humano: uma abordagem de avaliação de risco e contribuição ao processo de atualização da legislação brasileira, eng sant ambiente, v. 14, n. 1, p. 68-78, 2009.

LAKATOS, E. M., MARCONI, M. A. Fundamentos de Metodologia Cientifica. 7 ed. Editora Atlas. 2010.

MARCHESAN, E. SARTORI. Gerson Meneghetti Sarzi. AVILA. Luis Antonio. MACHADO. Sérgio Luiz de Oliveira. ZANELLA. Renato. PRIME. Ednei Gilberto, MACEDO. Vera Regina Musso. MARCHESAN. Marcos Garcia. Resíduos de agrotóxicos na água de rios da Depressão Central do Estado do Rio Grande do Sul, Brasil. Ciência Rural, Santa Maria, v.40, n.5, p.10531059, mai, 2010

MINAYO. S. Técnicas e Metodologia de Pesquisa. 10 ed. Editora LTR. 2008.

MINISTÉRIO DA SAÚDE. Boletim Epidemiológico Secretaria de Vigilância em Saúde, Volume 44, n $^{\circ} 17$ - 2013, Monitoramento de Agrotóxicos na Água para Consumo Humano no Brasil, 2011/2012.

SIQUEIRA, L. P.; SHINOHARA, N. K. S.,LIMA, R. M.T., PAIVA, J. E., FILHO, J. L. L., CARVALHO, I. T. Avaliação microbiológica da água de consumo empregada em unidades de alimentação. Ciência e saúde coletiva, v. 15, n. 1, p. 63-66. 2010 . 
STEFFEN, Gerusa Pauli Kist. STEFFEN, Ricardo Bemfica. ANTONIOLLI. Zaida Inês. Contaminação do solo e da água pelo uso de agrotóxicos. Tecnologia Ambiental. v. 15, n. 1, 2011.

SOARES, W.; ALMEIDA, R. M. V. R.; MORO S. Trabalho rural e fatores de risco associados ao regime de uso de agrotóxicos em Minas Gerais, Brasil. Caderno de Saúde Pública, v. 19, n. 4, p. 1.117-1.127, jul-ago/2003.

VEIGA, M. M.; SILVA, D. M.; VEIGA, L. B. E.; FARIA, M. V. C. Análise da contaminação dos sistemas hídricos por agrotóxicos numa pequena comunidade rural do Sudeste do Brasil. Caderno de Saúde Pública.vol.22 nº.11 Rio de Janeiro, p. 2391- 2399, Nov/2006

\section{Como citar este artigo (Formato ABNT):}

ALMEIDA NETO, M.G.; REIS, R.B.S. Agrotóxicos em água para o consumo humano: revisão de literatura. Id on Line Revista Multidisciplinar e de Psicologia, Janeiro de 2017, vol.10, n.33, p. 274-282. ISSN: 1981-1179.

Recebido: 06/12/2016

Aceito: 08/12/2016 